\title{
An Investigation of How Pixel Size Affects the Energy Resolution of a Photon Counting X-ray Imaging System
}

\author{
Muhammad Masood \\ Sarfaraz and Aamir Khan \\ COMSATS Institute of IT, \\ WahCantt. Pakistan.
}

\author{
Farah Batool \\ GC University Faisalabad, \\ Pakistan
}

\author{
Sheheryar Khan \\ COMSATS Institute of IT, \\ WahCantt. Pakistan.
}

\begin{abstract}
Radiation imaging sensors are being used in a variety of applications and a similar kind of sensor, namely the "Timepix", has been developed. The aim of this paper is to study and analyze its behavior and discover new technique that can be further integrated into the next version of the sensor in order to enhance its functionality. In this regard, the effect of charge sharing between the pixels has been studied as an initial starting point and an attempt has been made to discover the center peak position by removing the effect of charge sharing.
\end{abstract}

Keywords: Radiation Imaging sensor, Timepix, Energy Spectrum.

\section{INTRODUCTION}

The digital revolution within technology has been made possible because of the efficiency and minimization of electronic circuits. State-of-the art chips have been designed whose most important features include low power consumption, high noise immunity and which operate on very high clock frequencies. These revolutions within the world of science have offered new avenues for micro electronics.Timepix is an excellent chip but, at present, it is still not ready to be used as a medical imaging sensor as it is still too small and only has the ability to handle low flux rates. At this point, more research is required to overcome these problems. In this paper we attempt to discover some better solution in order to enhance the features of the present Timepix. In order to achieve, analysis has been performed using different metals. The tests and the analysis have been conducted using MATLAB. The key point is to discover a method which will provide the best possible results.

It is hoped that the results thus obtained can then be integrated into a future version of the Timepix chip and thus produce improved results.

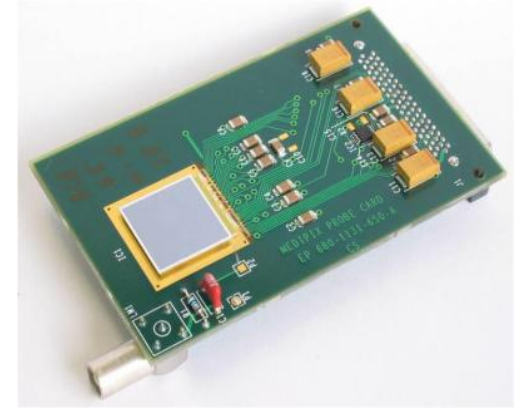

Fig 1: TIMEPIX CHIP [1]

\section{THEORY AND SYSTEM DESIGN}

Radiation is defined as the energy that comes from a source and travels through space or a medium and the energy which travels through space or a medium absorbed in another body [2], [3].Radiation can be categorized as given below Charged Particulate Radiation

- Fast Electron (Beta Decay)

- Heavy charged Particles (Alpha Decay) Uncharged Radiation

- Electromagnetic Radiation (Gamma Rays Following Beta Decay)

- Neutrons

Radioactivity is the process through which unstable atomic nuclei spontaneously emit subatomic particles.During this process it emits alpha particles, beta particles, gamma rays or electromagnetic rays.

The decay equation for radioactivity decay is defined as the rate of decay of the radioactive nuclei in a large sample that depends only on the number of decaying nuclei in the sample. A mathematical expression for the decay equation can be written as

$$
\frac{d N}{d t} \mid \operatorname{dec} a y=-\lambda N
$$

Where $d N$ is the number of radioactive nuclei in the time window $d t$ and $\lambda$ is the decay constant.The first and original unit of radioactivity was the Curie $(\mathrm{Ci})$ which is defined as

$$
1 \text { curie }(\mathrm{Ci})=3.7 \times 10^{10} \text { disintegration/second }
$$

The Curie is not used as the standard SI unit. It is replaced by the SI standard unit, the Becquerel (Bq).

$$
1 \mathrm{~Bq}=2.703 \times 10^{-11} \mathrm{Ci}
$$

For the measurement of radiation energy the traditional unit is the electron volt (ev).

$$
1 \mathrm{ev}=1.6 \times 10-{ }^{19} \mathrm{~J}
$$

The energy and frequency can be related as follows

$$
\mathrm{E}=\mathrm{hv}
$$

where $h$ is Planks constant and $f$ is the frequency and is measured in electron volts (ev) and the wavelength $\lambda$ related to the photon energy is measured in meters $(\mathrm{m})$.

$$
\lambda=\frac{1.240 \times 10^{-6}}{\mathrm{E}}
$$

Two types of beta decay are the electron emission $\left(\beta^{-}\right)$and the positron emission $\left(\beta^{+}\right)$. 
The decay process can be written schematically as

$$
{ }_{Z}^{A} X \rightarrow{ }_{Z+1}^{A} Y+\beta^{-}+\bar{v}
$$

in which $\mathrm{X}$ and $\mathrm{Y}$ are the initial and final nuclear species; $\beta$ shows the electron emission and $\bar{v}$ is an antineutrino. The neutrino and antineutrino have very small interactions because they travel at the speed of light and are thus undetectable and are also invisible [2], [3].

Alpha decay occurs in the heaviest nuclides. The decay process is written schematically as

$$
{ }_{Z}^{A} X \rightarrow{ }_{Z-2}^{A-4} Y+{ }_{2}^{4} \alpha
$$

The majority of the energy for the alpha particles remains between 4 and $6 \mathrm{Mev}$. However, because of the dependency with regards to the half - life of the energy process there are minor variations in the energy [2], [3]. Gamma rays are produced in alpha or beta rays because of their excitation or de-excitation. The example below in figure 2 shows the decay scheme for ${ }^{60} \mathrm{CO}$ for gamma rays sources. How do alpha or beta particles work in the excitation or de excitation states for gamma rays? When an alpha or beta ray is emitted from the nucleus the daughter nucleus goes into an excited state. On return to a lower energy state due to the de-excitation, then gamma rays are emitted.

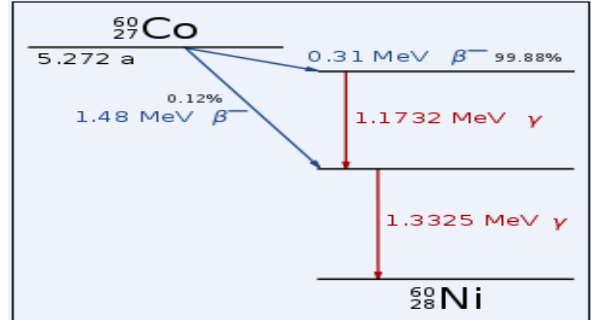

Fig. 2 Decay Scheme of CO [4]

When electrons receive some energy they go into the excitation state but this state will only exist for a short period of time. It is a natural characteristic of an electron that it returns to its ground state within a given timescale, which is based on the characteristics of the electron. The energy liberated in the transition from the excited to the ground state takes the form of a characteristic x-ray photon whose energy is given by the energy difference between the initial and final states. If the vacancy is created in the $\mathrm{k}$ shell then the outer shell falls into this and fill this vacancy. Vacancies created in the outer shells by the filling in of the $\mathrm{k}$ shell vacancies are filled by means of the emission of L-, M-,...series characteristic X-rays. When an electron is removed from an atom and thus leaving a vacancy, an electron from the outer energy level falls into the vacancy. The electron then falls from the outer level due to the release of energy or the emission of a photon.

Energy can also be transferred to another electron, which is ejected from the atom. This ejected electron is called an auger electron. Figure 3 below shows a characteristic X-ray and auger electron.

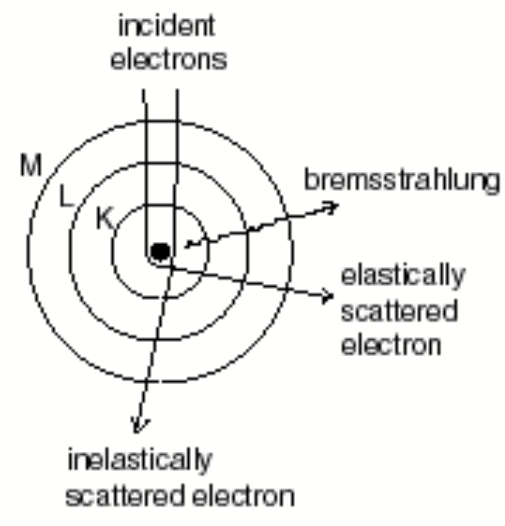

Fig. 3: Showing the production of bremsstrahlung, characteristic X-rays, and Auger electrons[5].
Neutron sources are generally stated as the emission of neutrons. There are several kinds of neutron sources

- Small Size Devices

- Medium Size Devices

- $\quad$ Large Size Devices

Measurement Setup is a hybrid silicon pixel device, the Timepix, is used. This device is the combination of two chips which are connected together by a bump-bonding method. One chip is a pixilated $300 \mu \mathrm{m}$ thick silicon detector chip and other is a bottom read-out chip with matrix dimensions of 256x256. This chip was developed at CERN by the MEDIPIX collaboration [7]. In this setup, fluorescence materials are placed in front of an x-ray radiation source to create fluorescence radiation and a detector is placed behind the source at a certain angle. A schematic of this setup is shown in figure 4 while the actual setup is shown in figure 5 below. 


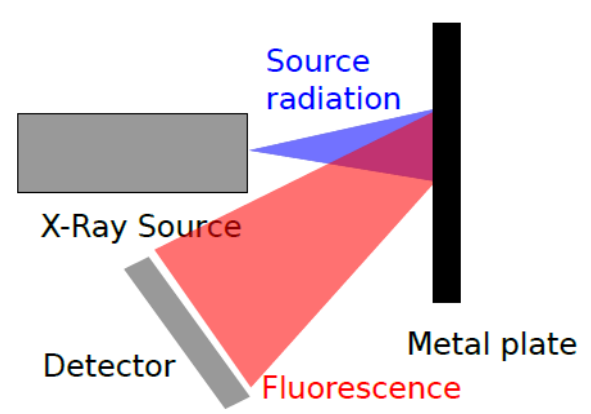

Fig.4: Measurement Setup[6]

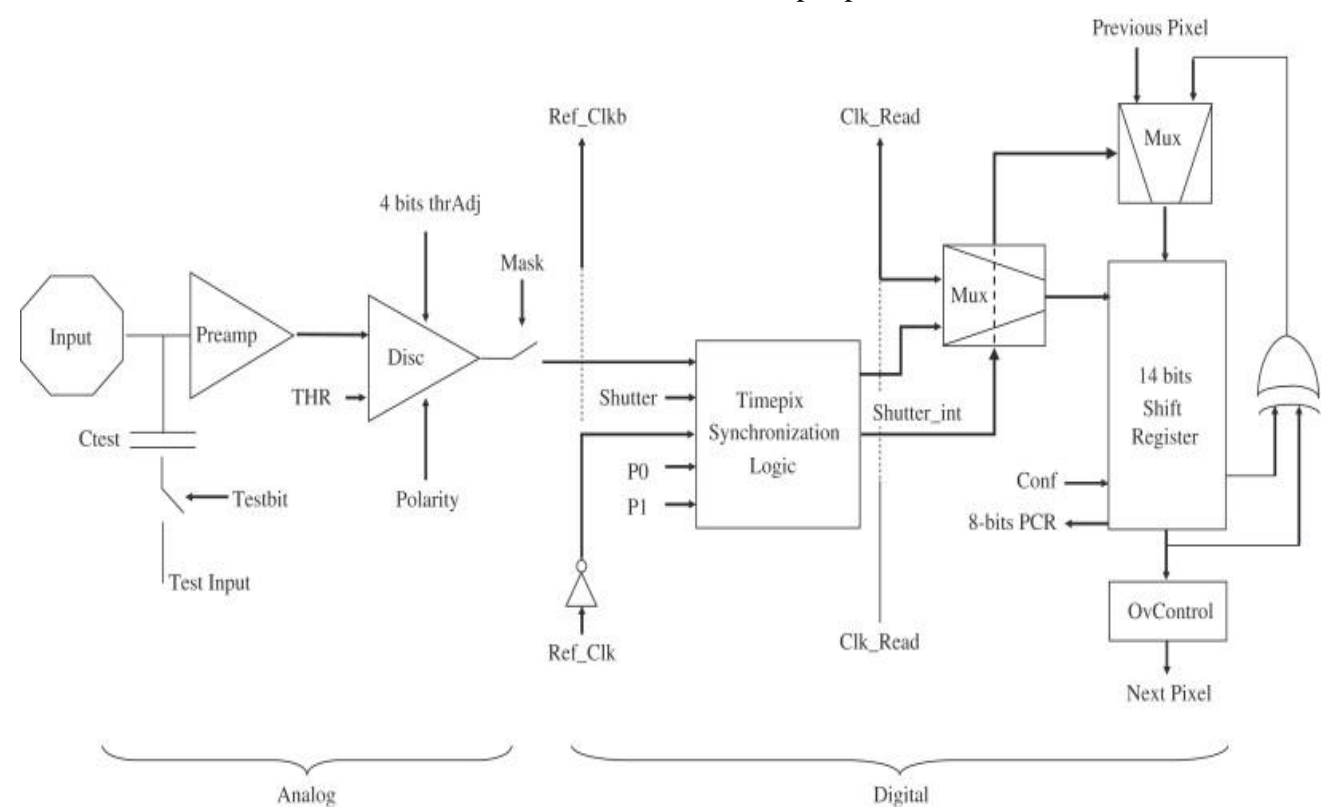

Fig. 6: Timepix pixel cell schematic[7]

Charge sharing-Cluster:The charge collected by each pixel in the cluster can be measured using the Timepix device. The charge collection speed depends on the applied bias voltage and the cluster size (number of pixels in the cluster) also depends on that voltage. The number of pixels in one cluster is called the cluster size. If measurements are performed without removing the cluster then the resulting spectrum is distorted. Thus a calibration of the Timepix is required in order to produce a satisfactory spectrum [10].If the computation of a cluster volume is conducted without any pixel calibration then the resulting spectrum is distorted.

Pixelman: The Institute of Experimental and Applied Physics (IEAP) at the Czech Technical University (CTU) in Prague have developed software for the MEDIPIX2 acquisition control and have named it Pixelman. This software supports all the MEDIPIX family devices including the MEDIPIX 2, 2.1, MXR, MEDIPIX2 MXR Quad, Timepix and Timepix Quad. All of this is achieved based on their internal flexibility.In order for these to operate for different options, it is necessary to use custom made plug-ins. Threshold equalization is used in order to compensate for the pixel to pixel threshold variations.

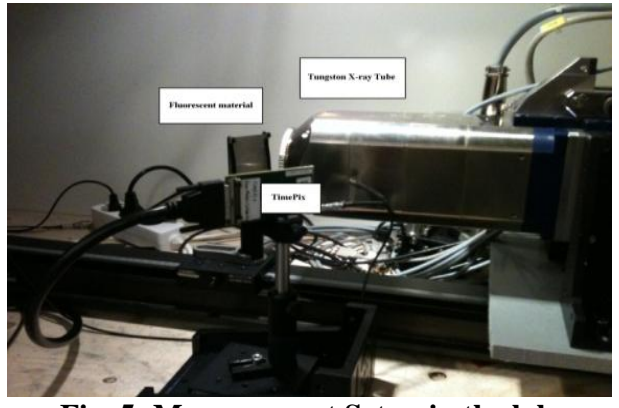

Fig. 5: Measurement Setup in the lab

Pixel cell: Although the architecture of MEDIPIX resembles that of the Timepix cell there are three features in the cell that differentiate the two. Figure 6 shows the schematics of the Timepix pixel cell.

The adjustment code is then selected for each pixel in order to make its threshold as near as possible to the average of the threshold distribution mean values. In other words it can be stated that threshold equalization is a procedure in which THL/THH adjustment is performed so as to make all the thresholds as homogenous as possible.Figure 7 below shows the threshold equalization of the TIMEPIX detector.

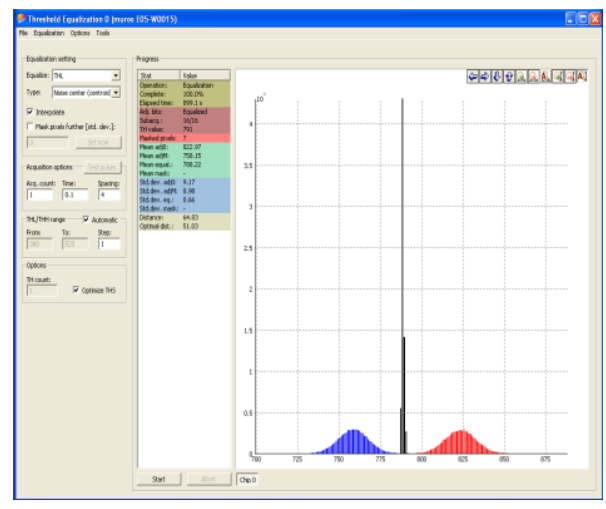

Fig. 7: Threshold Equalization of Timepix Detector 
Time over Threshold calibration: Before it is possible to make any measurements of metals, the TIMEPIX detector must be calibrated.

For this purpose the TIMEPIX will be used in Time-overThreshold (TOT) mode in which a single pixel is used for the calibration. The time -over-threshold will calibrate the energy.The calibration technique used in this case is different to that of the technique given in the TIMEPIX home page [8]. In this case, the maximum peaks of the fluorescence materials are used and these are then used for the calibration. A Gaussian curve fitting method is used in order to determine the maximum peak of the fluorescence materials. Figure below displays the TOT calibration curve

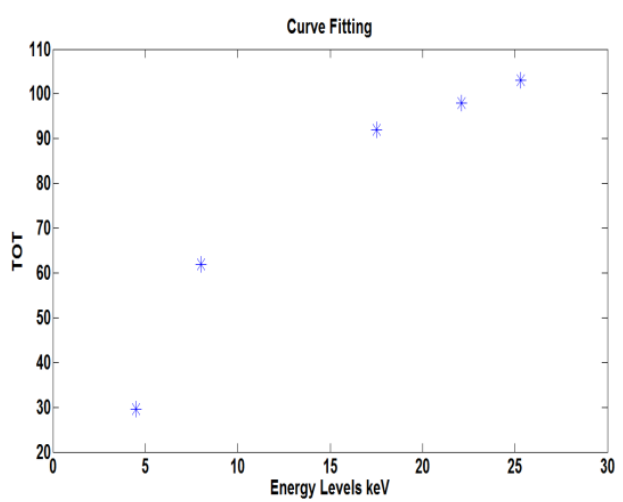

Fig.8: TOT Calibration measuerment

The mathematical equation below is used in order to verify the calibration

$$
\begin{gathered}
y=a x+b-\frac{c}{x-t} \\
y(x-t)=a x(x-t)+b(x-t)-c \\
y x-y t=a x^{2}-a x t+b x-b t-c \\
a x^{2}+b x-a x t-y x-b t+y t-c=0 \\
a x^{2}+(b-a t-y) x-b t+y t-c=0
\end{gathered}
$$

It is known that the general form of a quadratic equation is

$$
a x^{2}+b x+c=0
$$

And that the quadratic Formula is

$$
x=\frac{-b \pm \sqrt{b^{2}-4 a c}}{2 a}
$$

By placing the values of $\mathrm{a}, \mathrm{b}$ and $\mathrm{c}$ into the quadratic formula

$$
x=\frac{-(b-a t-y) \pm \sqrt{(b-a t-y)^{2}-4 a(-b t+y-c)}}{2 a}
$$

the equation thus becomes $=\frac{-(b-a t-y) \pm \sqrt{\left.b^{2}+a^{2} t^{2}+y^{2}-2 b a t-2 b y+2 a t y+4 a b t-4 a t y-4 a c\right)}}{2 a}$

and finally the calibration.

$$
x=\frac{t a+y-b+\sqrt{(b+t a-y)^{2}+4 a c}}{2 a}
$$

Basic Design Model: The purpose of this model is to develop a system which shows how the pixel size affects the energy resolution of photon counting $\mathrm{x}$-ray systems. The basic design model for the task is given in figure below. Radiation comes from an x-ray source and the hits come from the different fluorescence materials (cu, mo, Ag and tin) and the radiation is absorbed in the TIMEPIX detector. In order to achieve a better absorption in relation to the radiation, the florescence material and the detector are placed in a line of sight. As the radiations are absorbed in the TIMEPIX detector they are passed to a PC as images. The purpose of this PC is to read the absorption by using the Pixelman software which has been installed into the PC. The readings for these images are saved in an ASCIIXYC.txt format. It is also possible to save the images in a different format of the Pixelman software but the advantage of the format which has been used is that it saves a great deal of memory and the data can be easily processed in MATLAB.After acquiring the ASCIIXYC.txt image, this data is processed in MATLAB. The results of the process data will be displayed as the output. The working of this problem can be better viewed by means of the basic block diagram.

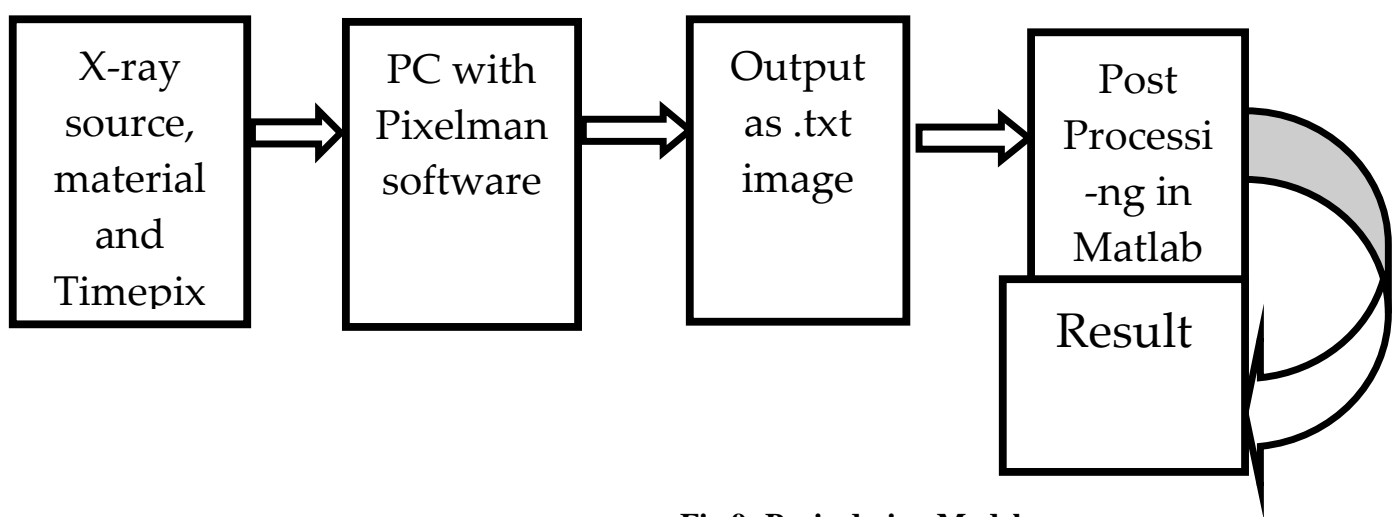

Fig.9: Basic design Model 
Response of pixel on certain energies: One thousand images have been taken for all the fluorescence materials $(\mathrm{Cu}, \mathrm{Mo}$, $\mathrm{Ag}$ and $\mathrm{Sn}$ ) in order and these have been provided with the proper settings as shown in figure 10 below, where the Acquisition Type is the frame. The acquisition count is 1000 which shows 1000 images of the different fluorescence materials $(\mathrm{Cu}, \mathrm{Mo}, \mathrm{Ag}$ and $\mathrm{Sn})$. The time taken for an image is 0.0005 . All the settings are fixed before the images are received in Pixelman which is attached to the measurement setup.

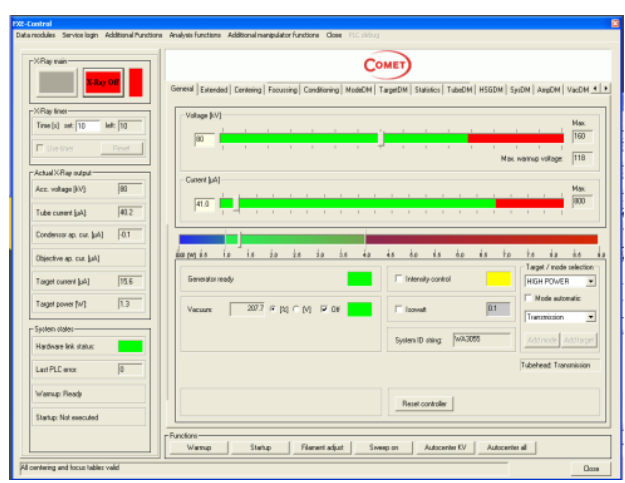

Fig.10: FXE control settings

Post Processing in MATLAB: The ASCIIXYC.txt image is processed in MATLAB according to the desired requirements. Only a pixel surrounded by zeros in a $3 \times 3$ pixel neighborhood is acceptable otherwise it should be deleted along with all the non-zero pixels in the $3 \times 3$ neighborhoods. This is because the requirement is to determine how the pixel responds to a particular energy.As the requirement is to calibrate each pixel individually it is very important that this deletion should take place. A signal is required from each pixel.If a signal comes from two pixels then the only information that is provided is that this is the energy of a photon but how much energy is from each of these pixels is unknown.
Only those pixels with a single hit are used for the calibration. MATLAB code scans images from different materials, pixels by pixel, in order to perform this action. The figure 11 below shows which pixels are deleted and which pixels are selected in the image on the basis of their neighboring pixels. A pixel value ' 1 ' represents a pixel having any value other than zero while a ' 0 ' pixel value naturally means a zero value for the pixel.

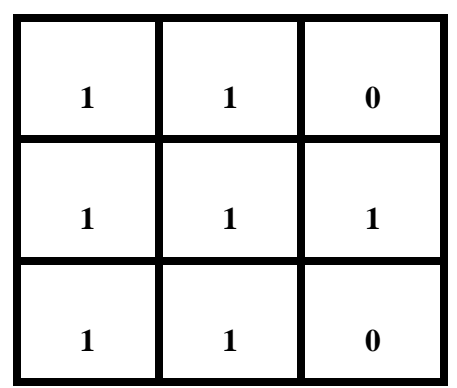

Fig.11: Pixel window, in which the center pixel is surrounded by some non-zero pixel values.The pixel and all the surrounding pixels in this case should be deleted/assigned a zero value.

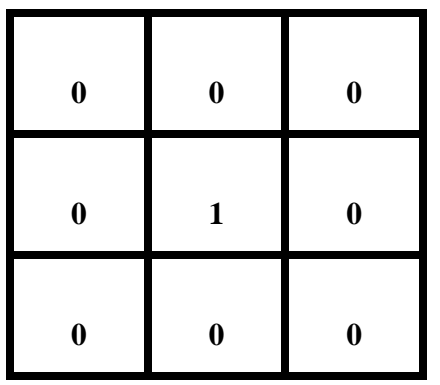

Fig.12: 3X3 Pixel window, in which the center pixel has all zeros in its neighborhood and it is the only one which is acceptable.

When the MATLAB code completely scans the entire ASCIIXYC.txt image it displays histograms of the different materials. These histograms are the number of hits against the time over threshold (TOT). Histograms of different materials show how pixels respond to the particular energies of the different materials. 
How do the pixels respond to particular certain energies? This has been performed by means of the acquisition of .txt images by using the measurement setup which is discussed above. These images are processed into the MATLAB code. This MATLAB code displays different results according to the given requirements. This code accepts those pixel values which are surrounded by zeros and deletes the remaining values. In other words, if the charge spreads over two pixels it is no longer of interest as the requirement is to discover how the pixels respond to particular energies.After the execution of the MATLAB code, figures 13 and 16 show single hits and the charge spread over two pixels for the fluorescence materials $(\mathrm{Cu})$ Copper and $(\mathrm{Sn})$ Tin. Then figures 14, and 17 shows that no charge spread exists over two pixels or no more double hits exist in these figures.Figures 15 and 18show the histograms for $(\mathrm{Cu})$ Copper and (Sn) Tin samples which are plotted for the number of pixels against the TOT (Time over Threshold).These histograms for these fluorescence materials show how the pixels respond to the different energy levels or TOT (Time over Threshold).

\section{RESULTS}

One photon of the copper sample delivered energy of $8.0 \mathrm{KeV}$ to one pixel. The histogram for the copper sample shows 0 to 255 energy levels and at these energy levels, different numbers of pixels exist. At the 62 photon energy level, a maximum number of hits are obtained.

1. Copper sample image result including single and double hits in figure 13 below.

2. Figure 14 shows that the double hits have been removed from the image because this is not a requirement in this case.

3. Figure 15 shows the histogram for the copper sample.

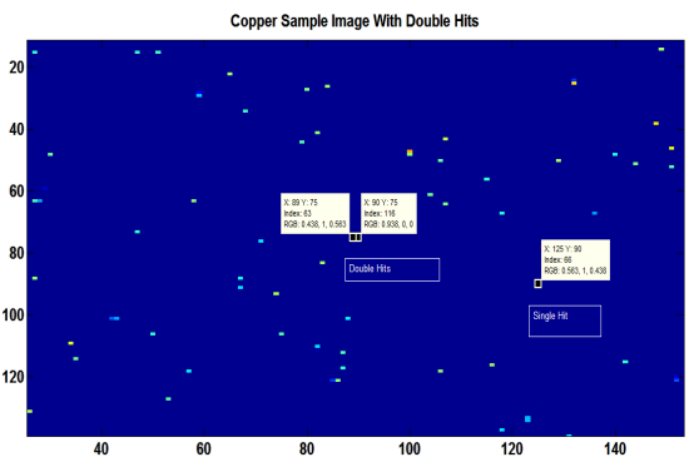

Fig. 13: Copper sample image displays the charge spread over two pixels

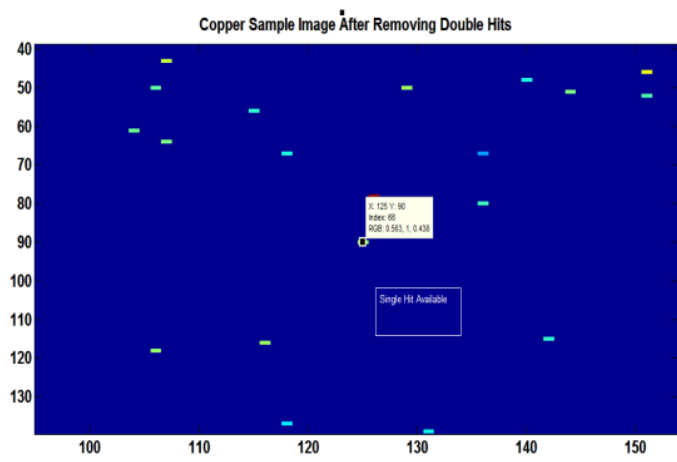

Fig. 14: copper sample image after removing double hits

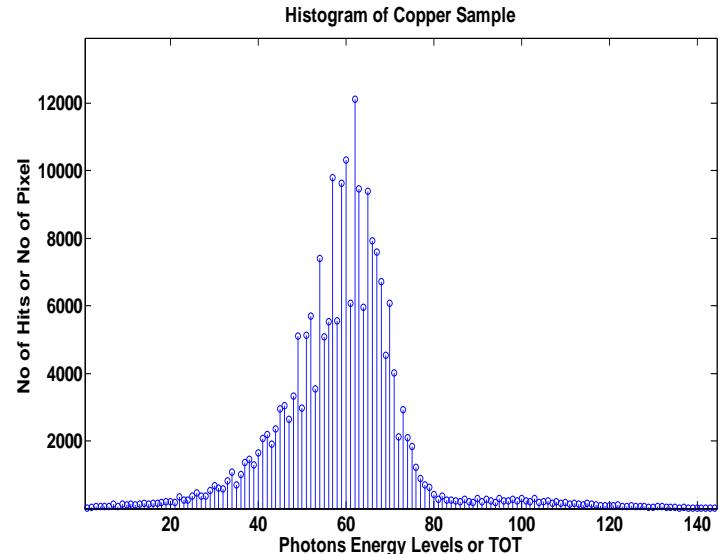

Fig. 15 Histogram for the copper sample

A photon of the tin sample delivered energy of $25.3 \mathrm{KeV}$ to one pixel. The histogram of the tin sample shows 0 to 255 energy levels and at these energy levels, different numbers of pixels exist. At the 103 photon energy level the maximumnumber of hits is obtained.

10. Tin sample image result, including the single and double hits, in figure 16 below.

11. Figure 17 shows that double hits have been removed from the image as these are not required in this case.

12. Figure 18 shows the histogram for the Tin sample.

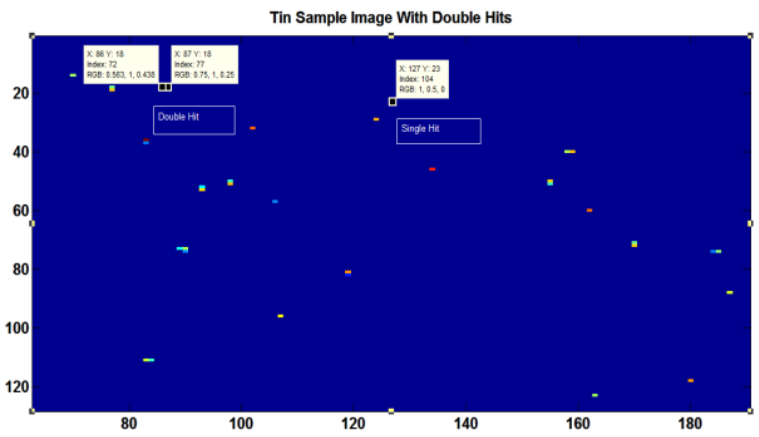

Fig. 16: Tin sample image with a charge spread over two pixels

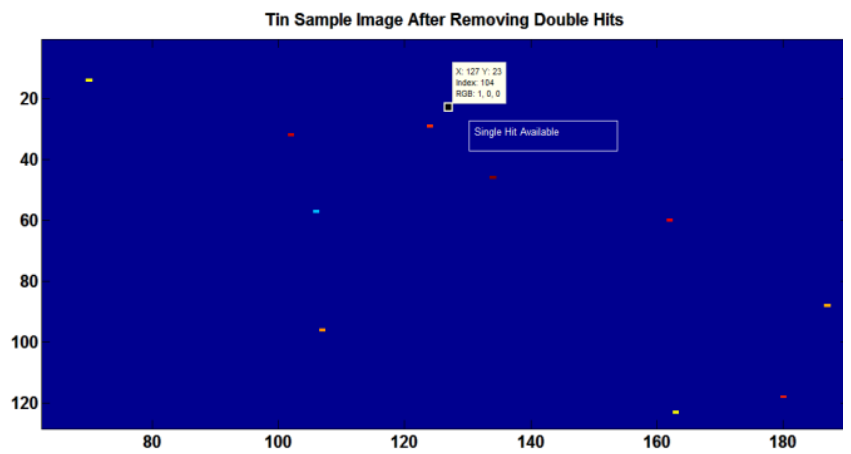

Fig. 17:Tin sample image after removing double hits 


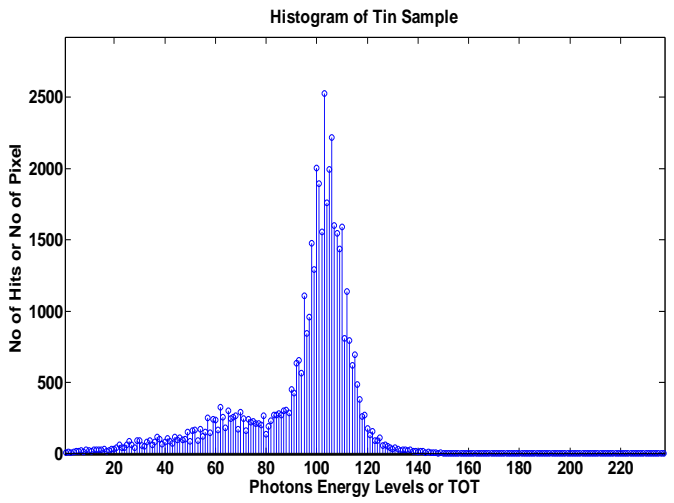

Fig. 18: Histogram for the Tin sample

\section{CONCLUSION}

System was successfully calibrated so that the response of the system could be observed for different metals at different energy levels.The analysis and the results thus generated during this work shows that the charge summing strategy implemented in MEDIPIX3 will successful for applications in this energy ranges. Timepix is used as tool to predict how MEDIPIX3 work and also test if sum over large area what will happen than. MEDIPIX3 and Timepix are similar but they are completely different. Spectrum of MEDIPIX3 is different from Timepix. Timepix can get the energy from every interaction in TOT mode but in MEDIPIX we get only count.

\section{REFERENCES}

[1] Victor Gutierrez Diez,'Measurements of time pix detectors performance', Universidad Complutense de Madrid, September 2010.

[2] Glenn F. Knoll, 'Radiation Detection and Measurement', ISBN 0-471-07338-5

[3] Syed Naeem Ahmed, 'Physics \& Engineering of Radiation Detection by', ISBN -10:0-12-045581-1

[4] Clinical Physics available at:http://www.jimoid.com/Physics/Clinical\%20Physics/c o60_decay.htm

[5] The Origin of Characteristic X-rays, available at http://www4.nau.edu/microanalysis/Microprobe/XrayCharacteristic.html

[6] Erik Fröjdh,'X-ray absorption and charge transport in a pixellatedCdTe detector with single photon processing read out', Mid Sweden University , Holmgatan 10,851 70 Sundsvall, Sweden .

[7] X. Llopart,' Timepix a $65 \mathrm{~K}$ programmable pixel readout chip for arrival time, energy and/or photon counting measurements', Volume 581, Issues 1-2,21 October 2007 , pages 485-494. VCL 2007-Proceedings of the $11^{\text {th }}$ International Vienna Conference on Instrumentation.

[8] Medipix in IEAP, available at: http://aladdin.utef.cvut.cz/ofat/Methods/TimePixCalibrat ion/index.htm 